ARTÍCULOS

CRÍTICA, Revista Hispanoamericana de Filosofía

Vol. XXVI, No. 78 (diciembre 1994): 3-26

\title{
CIRCULARIDADES EN LA CONTRASTACIÓN EXPERIMENTAL
}

\author{
JULIÁN GARRIDO GARRIDO \\ Facultad de Ciencias \\ Universidad de Granada
}

\section{Introducción}

Las leyes de la ciencia factual son enunciados generales cuya verdad empírica se evalúa investigando sus correspondencias con los hechos. El carácter universal irrestricto de sus dominios de validez imposibilita la exhaustividad de sus comprobaciones empíricas. En consecuencia, las contrastaciones efectivas de las leyes se realizan mediante algunas de sus consecuencias, cuyas formas son las de predicciones de datos singulares. Pero las predicciones contrastables de las leyes no consisten nunca en su simple singularización respecto a la situación particular de interés, sino que se deducen de esa ley singularizada junto con otros enunciados empíricos referidos a esa misma situación. Estos enunciados auxiliares son muy variados: datos de las propiedades o condiciones en que se encuentra el objeto físico de la predicción, datos de constantes físicas y, en la mayoría de los casos, otras leyes pertinentes para el fenómeno, también singularizadas respecto a éste. Por ejemplo, la predicción de la velocidad del sonido en el aire es consecuencia conjunta de (a) las leyes del movimiento de Newton, de la conser- 
vación de la masa y de la ecuación de estado adiabática de los gases, aplicadas al fluido vibrante, y de (b) los datos del coeficiente adiabático del aire, de la presión atmosférica y de la densidad del aire, aplicados a dicha presión y a la temperatura considerada. En general, las predicciones contrastables de las leyes nunca son consecuencias exclusivas de una ley aislada, sino que se deducen de conjuntos de enunciados.

Por otro lado, las predicciones de las leyes de la ciencia factual madura son generalmente cuantitativas, expresan el valor numérico esperado de ciertas magnitudes. Su contrastación requiere mucho más que la simple observación, exige la medición del valor de esas magnitudes en situaciones experimentales que reproduzcan materialmente la situación teórica expresada por la ley o las leyes contrastadas en el despliegue de la predicción. Pero la medición de magnitudes es predominantemente indirecta. Los instrumentos realizan usualmente medidas directas de longitudes o ángulos, las cuales, en virtud de leyes científicas fundamentadoras del aparato, son proporcionales a los valores de la magnitud de interés. Esta proporcionalidad permite graduar la escala del medidor en términos de la magnitud que se mide indirectamente y leer "directamente" sus valores. Por ejemplo, la intensidad de la corriente eléctrica, medida indirectamente en un amperímetro mecánico, es proporcional al ángulo indicado por la aguja del aparato, como consecuencia conjunta de las leyes del movimiento de Newton, de la fuerza de Lorentz y de Hooke. En definitiva, aunque muchos medidores permiten leer "directamente" bastantes mediciones indirectas, no debe ignorarse que éstas se fundamentan en leyes científicas factuales.

Las consideraciones anteriores expresan las dos características más significativas de la contrastación experimental de las leyes de la ciencia factual madura: "el holismo o globalismo en la generación de predicciones" (las predic- 
ciones contrastables de las leyes no se deducen exclusivamente de ellas, sino de conjuntos más amplios de enunciados) y "la carga teórica de las mediciones" (las mediciones contrastadoras de las leyes se fundamentan, a su vez, en leyes). Ambas características fueron señaladas y analizadas minuciosamente por Pierre Duhem en La théorie physique, son objet et sa structure (1905). La filosofía analítica de la ciencia, desarrollada posteriormente, las ha convertido en tópicos o lugares comunes, respetando en esencia las consideraciones originales del físico francés. Prueba de ello es la frecuente denominación "problema de Duhem" para designar el problema metodológico planteado como consecuencia del "holismo": cuando las predicciones teóricas son refutadas por mediciones bien fundamentadas, al ser las predicciones consecuencias de conjuntos de enunciados que suelen incluir una o varias leyes y diversos datos de las condiciones presentes, es preciso decidir qué premisas de la predicción deben ser rechazadas como consecuencia de la refutación. La solución del "problema de Duhem" consistirá en la especificación de los criterios que deberían regular las decisiones de rechazo o modificación más adecuadas para el desarrollo del conocimiento científico.

El "problema de Duhem" es generado por el "holismo" y constituye un problema real. En estos casos, los científicos activos combinan consideraciones conservadoras del conocimiento cuestionado (las modificaciones que hay que realizar se ensayan en las premisas predictivas poco generales antes que en las leyes o principios teóricos implicados) y consideraciones críticas (las modificaciones propuestas son aceptadas como concluyentes si se demuestra su carácter no ad hoc, si son contrastadas independientemente de modo satisfactorio), junto con una fuerte dosis de paciencia (muchas refutaciones no son resueltas en el momento de su descubrimiento y constituyen durante cierto tiempo pro- 
blemas por resolver). En cualquier caso, se trata de un problema manifiesto en la práctica científica.

Por otro lado, la otra característica de la contrastación experimental, "la carga teórica de las mediciones", implica, cuando éstas son utilizadas como contrastadoras (no se olvide que las mediciones tienen otras funciones, cognoscitivas y prácticas, aunque aquí no se consideren), el fenómeno denominado "circularidades en la contrastación experimental": las leyes y las teorías científicas se contrastan comparando algunas de su predicciones con mediciones, las cuales, a su vez, se apoyan en leyes y teorías científicas. Este tipo de circularidades ha conducido a toda clase de opiniones en el ámbito de la filosofía de la ciencia: desde escepticismos radicales respecto a la posibilidad de la experiencia para controlar las teorías, hasta concepciones más ajustadas a la práctica científica real, desde la cual puede ponerse en evidencia que las teorías no se autoconfirman y que, en consecuencia, no se producen círculos viciosos. Es significativo, sin embargo, que este debate filosófico no tiene correspondencia con ningún problema metodológico específico en el ámbito de la práctica científica: los científicos no deben tomar decisiones especiales de ningún tipo cuando realizan contrastaciones en las que hay leyes que se repiten en las deducciones de la predicción y de la medición que se confrontan.

En resumen, "el holismo de las predicciones" da lugar al "problema de Duhem", manifiesto en la práctica científica, pero "la carga teórica de las mediciones", aunque implica "circularidades en la contrastación experimental", no conduce a una problemática metodológica específica, distinta y paralela al "problema de Duhem". En los próximos apartados analizaré el fenómeno de las circularidades y argumentaré que, en el aspecto metodológico, contribuye simplemente a reforzar el "holismo" y, en consecuencia, a engrosar el "problema de Duhem". 


\section{Circularidades no autoconfirmantes}

No es difícil entender por qué las circularidades mencionadas no producen autoconfirmaciones. En primer lugar, debe considerarse que los conocimientos teóricos contrastados y los contrastadores no coinciden. Si se hacen explícitas las deducciones que conducen a la predicción y a la medición, respectivamente (véase Bunge, 1967 y 1973, donde se encuentran los análisis más detallados que conozco de la contrastación experimental) se debe concluir que las premisas de la predicción y las premisas de la medición son conjuntos diferentes de enunciados, aunque su intersección no suele ser vacía. A esta primera circunstancia se debe unir la siguiente: en la práctica científica real no se cuestionan simultáneamente todos los conocimientos utilizados en un momento dado. La comprobación de las predicciones de determinadas leyes se realiza con mediciones obtenidas con ayuda de otras leyes, fundamentadoras de los instrumentos de medida utilizados, que en esa circunstancia se consideran adecuadas porque en otros momentos han sido sometidas, a su vez, a contrastación experimental. De estas consideraciones se desprende la inexistencia de la autoconfirmación y que, en última instancia, lo único relevante es el "problema de Duhem": las leyes contrastadas lo manifiestan en el curso de sus contrastaciones, y las leyes contrastadoras en aquellos otros momentos en que fueron sometidas a contrastación. Los análisis de Bunge mencionados antes están, en mi opinión, en la línea de estas conclusiones.

Sin embargo, las consideraciones anteriores parecen debilitarse si el solapamiento entre las premisas de la predicción y de la medición es apreciable. En particular, si una ley es corresponsable (junto con otros enunciados) de una predicción y, simultáneamente, es fundamento de una medición que la contrasta, entonces parece que suponerla verdadera en la medición (porque en otros momentos se 
sometió a contrastación) obliga a considerarla verdadera en la predicción. En otras palabras, las contrastaciones en las que una misma ley se repite en la predicción y en las mediciones parecen incapaces de refutar dicha ley. Considérense, a título de ejemplo, contrastaciones de la ley de Ohm utilizando amperímetros y voltímetros, y que estos últimos se fundamentan teóricamente en varias leyes entre las que se encuentra la propia ley de Ohm. Es importante percibir que estas contrastaciones pueden revelar discordancias entre predicción y medición, puesto que éstas no se deducen simplemente de la ley que se repite (recuérdese el "holismo"), pero da la sensación de que en caso de fracaso predictivo no podría considerarse sospechosa dicha ley en la búsqueda de los responsables. En definitiva, aunque este tipo de contrastaciones tampoco autoconfirma las teorías, parecería incapaz de afectar determinadas leyes de esas teorías; produciría autoconfirmación parcial.

Aunque la conclusión anterior fuese correcta, en absoluto justificaría un escepticismo frente al poder controlador de la experiencia. Implicaría simplemente que tal poder sería algo menor del supuesto en una concepción del experimento científico que fuese inconsciente de sus circularidades. Pero resulta que la conclusión obtenida no es cierta. Para mostrarlo, desarrollaré un ejemplo de contrastación experimental en el que hay una ley que se repite en la predicción y en la medición, y en el que dicha ley resulta refutada. La contrastación del efecto Venturi mediante tubos piezométricos utiliza la ley irrotacional de Bernoulli tanto en la predicción como en la medición de dicho efecto. La contrastación conduce a la refutación, lo que pone en evidencia su carácter no autoconfirmante. Pero, además, la responsable de la refutación resulta ser la ley irrotacional de Bernoulli, pese a su doble presencia en la predicción y en la medición. En definitiva, la circularidad en la con- 
frontación teoría-experimento no salva de la experiencia ni siquiera a las leyes responsables de dicha circularidad. El análisis detallado de esta contrastación permitirá comprender por qué no se producen autoconfirmaciones parciales y cómo lo que resulta únicamente es un reforzamiento del "holismo".

\section{Contrastación del efecto Venturi}

El efecto Venturi consiste en variaciones de presión entre puntos de un fluido perfecto e incompresible, con movimiento estacionario e irrotacional en el campo gravitatorio terrestre, cuando atraviesa tubos de corriente de velocidad de sección variable. Esas variaciones de presión pueden medirse mediante tubos piezométricos verticales situados sobre la tubería horizontal que materializa los tubos de corriente de velocidad en los que se produce el efecto. Las predicciones y las mediciones de presión que constituyen su contrastación se obtienen mediante principios teóricos y leyes experimentales pertinentes para esta situación ideal, que en el experimento se intenta reproducir lo más aproximadamente posible. Los principios teóricos que intervienen son tres: las leyes del movimiento y de la gravitación de Newton y la ley de la conservación de la masa, adaptadas a un elemento diferencial del volumen del fluido concebido como medio continuo. Las leyes experimentales pertinentes son también tres: la ley de Torricelli, la ley de Bernoulli (irrotacional) y la ecuación de continuidad (incompresible e irrotacional) en tubos de corriente. Las leyes de Torricelli y de Bernoulli son consecuencias, en condiciones de restricción diferentes, de las leyes del movimiento y de la gravitación; mientras que la ecuación de continuidad es consecuencia de la ley de la conservación de la masa. El análisis completo de la contrastación del efecto Ventu- 
ri debe remontarse a los principios teóricos, pues sólo de este modo las condiciones de aplicación de las leyes experimentales pertinentes resultan perfectamente explícitas. Para mayor claridad, las deducciones de las leyes experimentales se situaron en los apéndices y se consideró, en este lugar, una descripción resumida de ellas.

Deducción de Bernoulli irrotacional*

Ley del movimiento (fluidos) + fuerza externa gravitatoria terrestre + fluido perfecto + movimiento estacionario + movimiento irrotacional + fluido incompresible, implican Bernoulli irrotacional:

Para todo par de puntos, $i$ y $j$, en un fluido perfecto e incompresible, con movimiento estacionario e irrotacional en el campo gravitatorio terrestre:

$$
P_{i}+\rho g h_{i}+\frac{1}{2} \rho v_{i}^{2}=P_{j}+\rho g h_{j}+\frac{1}{2} \rho v_{j}^{2} .
$$

(Si el movimiento del fluido no fuese irrotacional resultaría la ley de Bernoulli, expresada por una ecuación idéntica a la anterior, pero aplicable únicamente a pares de puntos de una misma línea de corriente de velocidad.)

Deducción de Torricelli*

Ley del movimiento (fluidos) + fuerza externa gravitatoria terrestre + fluido en reposo + fluido incompresible, implican Torricelli:

Para todo par de puntos, $i$ y $j$, en un fluido incompresible en reposo en el campo gravitatorio terrestre:

$$
P_{i}+\rho g h_{i}=P_{j}+\rho g h_{j} .
$$

* Véase apéndice I, p. 22.

* Véase apéndice II, p. 23. 


\section{Deducción de continuidad*}

Conservación de la masa (fluidos) + fluido incompresible + tubo de corriente + movimiento irrotacional (velocidad constante en secciones transversales del tubo de corriente), implican continuidad (incompresible e irrotacional) en tubos de corriente de velocidad:

Para todo par de puntos, $i$ y $j$, en un fluido incompresible, con movimiento irrotacional a través de tubos de corriente de velocidad (con secciones transversales $S$ ):

$$
v_{i} S_{i}=v_{j} S_{j} .
$$

(Si el movimiento no fuese irrotacional, la velocidad no sería constante en las secciones $S$ y la ecuación de continuidad tendría la forma $\int v \cdot d s=$ cte, en lugar de $v S=$ cte.)

Una vez fijadas con precisión las condiciones de validez de estas leyes, pueden analizarse sus singularizaciones en la predicción y la medición de presiones en el efecto Venturi.

\subsection{Predicción del efecto Venturi}

La realización del experimento con agua, atravesando una tubería de sección variable en la superficie terrestre, con un caudal (volumen por unidad de tiempo) de entrada de líquido constante y no excesivamente grande, asegura un cumplimiento aproximado de las condiciones ideales: fluido perfecto e incompresible, con movimiento estacionario e irrotacional en el campo gravitatorio terrestre a través de tubos de corriente de velocidad. En estas circunstancias son aplicables (véanse apéndices I y III) la ley irrotacional de Bernoulli y la ecuación de continuidad (incompresible e irrotacional) en tubos de corriente de velocidad.

* Véase apéndice III, p. 24. 
Estas dos leyes, singularizadas en un mismo par de puntos, 1-2 (figura 1) - y considerando que $h_{1}=h_{2}$ y que, por conservación de la masa, $v S=V / t=C$ (caudal)-, conducen a:

$$
\begin{array}{ll}
P_{i}+\frac{1}{2} \rho v_{1}^{2}=P_{2}+\frac{1}{2} \rho v_{2}^{2} & \text { (Bernoulli irrotacional) } \\
v_{1} S_{1}=v_{2} S_{2}=C & \text { (continuidad), }
\end{array}
$$

y resulta así la predicción del efecto Venturi:

$$
P_{1}-P_{2}=\frac{1}{2} \rho C^{2} \frac{S_{1}^{2}-S_{2}^{2}}{S_{1}^{2} \cdot S_{2}^{2}} \quad \text { (predicción), }
$$

que indica disminuciones de presión en los estrechamientos y aumentos en los ensanchamientos.

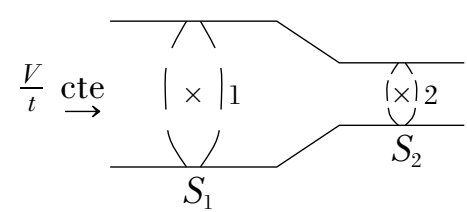

Figura 1

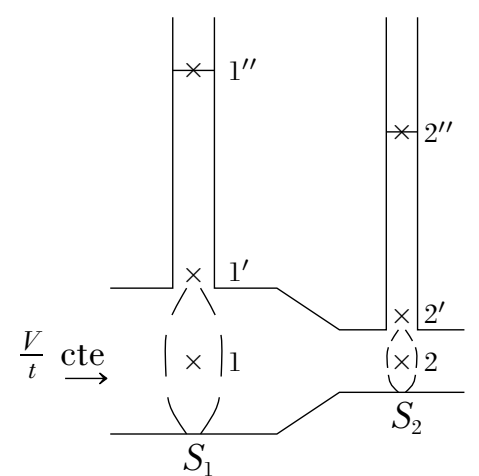

Figura 2

\subsection{Medición del efecto Venturi}

Las presiones del efecto Venturi pueden medirse con tubos piezométricos verticales colocados sobre la tubería horizontal en los lugares de interés (figura 2). En los tubos verticales el líquido está en reposo y resulta aplicable la ley de 
Torricelli (apéndice II); y en los puntos de la superficie está a la presión atmosférica. En la tubería horizontal, al haber movimiento estacionario e irrotacional, sigue siendo aplicable la ley irrotacional de Bernoulli (apéndice I). Además, los pares de puntos bajo un mismo piezómetro, al pertenecer a la misma sección transversal del tubo de corriente, tienen (por ser el movimiento irrotacional) la misma velocidad y se simplifica para ellos la expresión de la ley de Bernoulli.

Singularizando la ley de Torricelli en $1^{\prime}-1^{\prime \prime}$ y en $2^{\prime}-2^{\prime \prime}$, y la ley irrotacional de Bernoulli en $1-1^{\prime}$ y en $2-2^{\prime}$, y considerando que $v_{1}=v_{1}^{\prime}$ y $v_{2}=v_{2}^{\prime}$, resulta:

$$
\begin{array}{ll}
P_{1}+\rho g h_{1}=P_{1^{\prime \prime}}+\rho g h_{1^{\prime \prime}} & \text { (Torricelli }+ \text { Bernoulli irrotac.) } \\
P_{2}+\rho g h_{2}=P_{2^{\prime \prime}}+\rho g h_{2^{\prime \prime}} & \text { (Torricelli }+ \text { Bernoulli irrotac.) }
\end{array}
$$

y, como $h_{1}=h_{2}$ y $P_{1^{\prime \prime}}=P_{2^{\prime \prime}}=P_{\text {at }}$, resulta la medición del efecto Venturi:

$$
P_{1}-P_{2}=\rho g\left(h_{1^{\prime \prime}}-h_{2^{\prime \prime}}\right) \quad \text { (medición) }
$$

según la cual, los descensos de altura piezométrica indican disminuciones de presión, y las elevaciones señalan aumentos de presión.

\subsection{Contraste entre predicción y medición}

La comparación entre las variaciones de presión predichas y medidas conduce a la siguiente relación cuantitativa entre magnitudes directamente medibles:

$$
\frac{1}{2} C^{2} \frac{S_{1}^{2}-S_{2}^{2}}{S_{1}^{2} \cdot S_{2}^{2}}=g\left(h_{1^{\prime \prime}}-h_{2^{\prime \prime}}\right) \quad(\text { contraste })
$$

la cual expresa que en los estrechamientos de la tubería 
horizontal deben reproducirse descensos, y en los ensanchamientos, elevaciones de las alturas piezométricas.

Los experimentos contrastadores de este efecto revelan discrepancias con este resultado. Superpuesto a él, se produce una disminución de presión, pequeña pero progresiva, a medida que el líquido recorre la tubería. Este fenómeno, denominado "pérdida de carga en tubería", es perfectamente apreciable cuando la sección de ésta es constante. En estas condiciones el efecto Venturi implica presión y altura piezométrica constantes a lo largo de la tubería, mientras que lo observado es una lenta disminución de dichas alturas. La razón de esto último es la pequeña viscosidad del agua, cuyos efectos no resultan despreciables. La responsabilidad de este factor es contrastable independientemente, pues, a igualdad de las restantes condiciones experimentales, cuanto más viscoso es el fluido de prueba, más elevada es la "pérdida de carga". Considerando, entonces, las leyes experimentales implicadas en la predicción y en la medición discordantes, y dado que (véanse los apéndices) ni la ley de Torricelli ni la ecuación de continuidad descansan en la hipótesis de fluido perfecto o no viscoso, es claro que la ley cuya aplicación se debe rechazar en esta circunstancia experimental es la irrotacional de Bernoulli. En definitiva, la doble presencia de una ley experimental en predicciones y mediciones de un experimento no elimina la posibilidad de contrastaciones negativas, ni de que el fragmento de teoría que se deba modificar sea la propia ley que se repite. Naturalmente, en muchos casos de contrastación experimental con circularidad similar a la aquí considerada, se pueden producir confirmaciones. $\mathrm{Y}$ otros casos pueden conducir a refutaciones superables por modificación de alguna de las leyes experimentales que no se repite. Pero el que puedan encontrarse casos como el presente es muestra significativa del carácter no vicioso o autoconfirmante, ni siquiera de modo parcial, de estas circularidades. 
4. Reformulación del esquema usual de contrastación experimental

La simple exposición de ejemplos favorables a una tesis no constituye prueba suficiente de ella. Pero la estructura de la contrastación analizada es fácilmente generalizable. Permite, por ello, encontrar respuesta adecuada a la cuestión general: ¿por qué los usos circulares de conocimiento en la contrastación no conducen, contra toda apariencia intuitiva, a autoconfirmación?

Ante todo, el análisis de la contrastación anterior aconseja distinguir circularidades o repeticiones en dos niveles: en los principios teóricos y en las leyes experimentales. (En el efecto Venturi, no sólo se repite, en la predicción y en la medición, la ley irrotacional de Bernoulli, sino también los principios teóricos de la ley del movimiento y de la ley de la gravitación de Newton.)

Sin embargo, la circularidad en los principios teóricos, por sí sola, no es preocupante. Los principios teóricos son tan generales que precisan condiciones de restricción o hipótesis de modelo que los particularizan en leyes experimentales (véanse los apéndices como tres ejemplos de ello) para poder generar predicciones contrastables. En suma, los principios se contrastan con leyes experimentales. En consecuencia, si un principio teórico interviene circularmente en una contrastación, se producen dos posibilidades. La más frecuente es que el principio se particularice hacia la predicción y hacia la medición mediante diferentes leyes experimentales. No hay, entonces, circularidad extrema pues pueden, en este caso, considerarse justificadas las leyes fundamentadoras de la medición porque en otro momento se sometieron a contrastación. La otra posibilidad, la generación de alguna ley experimental repetida en la predicción y en la medición (ejemplificada en el efecto Venturi), conduce a circularidad en el nivel de las leyes experimenta- 
les. Por lo tanto, la circularidad en los principios teóricos es, en sí, metodológicamente irrelevante (lo mismo sucede con la distinción estructuralista - Sneed, 1971 - entre conceptos $T$-teóricos y $T$-no teóricos, inspirada en ella); sólo es significativa la circularidad en las leyes experimentales.

Respecto a la circularidad en las leyes experimentales se impone la siguiente consideración. La deducción de predicciones y mediciones requiere singularizar las leyes pertinentes respecto a las circunstancias experimentales del caso. Como la predicción y la medición constituyen aspectos parciales diferentes de la compleja situación experimental, en el caso de que una misma ley experimental intervenga en ambas, lo hará singularizada en circunstancias diferentes; y es perfectamente posible, en principio, que una singularización de la ley sea correcta y no lo sea la otra. (Por ejemplo, en el efecto Venturi interviene la ley "irrotacional de Bernoulli", en pares de puntos cualesquiera, singularizada en horizontal para la predicción y en vertical para la medición. Sin embargo, con fluido perfecto y movimiento estacionario pero no irrotacional, lo aplicable hubiese sido la ley de "Bernoulli", con la misma ecuación matemática que la anterior, pero sólo válida en pares de puntos de una misma línea de corriente, o sea, singularizable en horizontal, pero no en vertical.) En suma, la "repetición" de una ley experimental para obtener una predicción y una medición no es tan absoluta si se considera que, para llegar a éstas, es preciso singularizarla respecto a situaciones particulares diferentes.

Mucho más contundente que las consideraciones anteriores (matizadoras y relativizadoras de las repeticiones que efectivamente se producen en las contrastaciones) es el siguiente argumento, que elimina toda apariencia viciosa de la circularidad (tanto de leyes como de principios) de la 
contrastación experimental. Se obtiene reestructurando el árbol deductivo de la contrastación experimental de un modo perfectamente legítimo desde un punto de vista lógico.

Metodológicamente, el científico compara predicciones y mediciones de magnitudes de todo tipo, y plantea problemáticas relativamente independientes para unas y otras. Casi toda la problemática de medición es esencialmente física. Sin embargo, su relevancia es tan grande para la contrastación de predicciones de teorías físicas, como para la de predicciones de las restantes ciencias químicas, biológicas o geológicas. Por este motivo, la construcción de medidores y la comprobación de predicciones son actividades generalmente separables. Desde esta perspectiva, la estructura de la contrastación del efecto Venturi ha sido expresada como: predicciones de presión (correspondientes a secciones en tuberías) comparadas con mediciones indirectas de presión (por medio de alturas piezométricas). Estas últimas, al basarse en alguna de las leyes de la predicción, entre otras, producen la apariencia de petición de principio o de circularidad. Así se refleja en el siguiente esquema:

Ley del mov. + Ley de la gravit. Conserv. de la masa Bernoulli irrotacional $\quad+\quad$ Continuidad

$$
P_{1}-P_{2}=\frac{1}{2} \rho C^{2} \frac{S_{1}^{2}-S_{2}^{2}}{S_{1}^{2} \cdot S_{2}^{2}}
$$

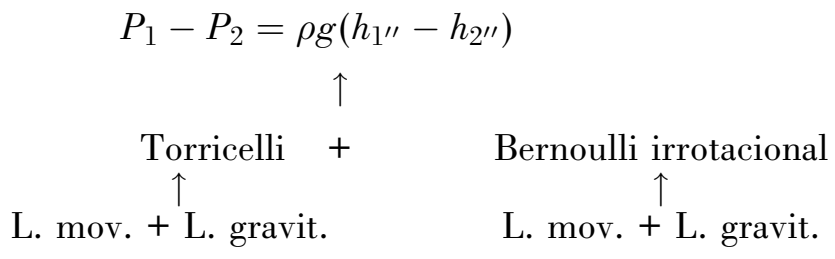


Sin embargo, desde un punto de vista lógico, el esquema anterior de la estructura de la contrastación es perfectamente reformulable del modo siguiente:

$$
\begin{aligned}
& \text { L.mov. }+\underset{\downarrow}{\text { L.grav. }} \quad \underset{\downarrow}{\quad \text { Cons.masa }} \quad \text { L.mov. }+\underset{\downarrow}{\text { L.grav. }} \quad \text { L.mov. }+\underset{\downarrow}{\text { L.grav } .} \\
& \text { Bernoulli irrot. + Continuidad Torricelli + Bernoulli irrot. } \\
& P_{1}-P_{2}=\frac{1}{2} \rho C^{2} \frac{S_{1}^{2}-S_{2}^{2}}{S_{1}^{2} \cdot S_{2}^{2}} \quad P_{1}-P_{2}=\rho g\left(h_{100}-h_{2^{\circ 0}}\right) \\
& \frac{1}{2} C^{2} \frac{S_{1}^{2}-S_{2}^{2}}{S_{1}^{2} \cdot S_{2}^{2}}=g\left(h_{100}-h_{2^{00}}\right)
\end{aligned}
$$

Mediciones directas

en el que predicciones de variaciones de altura piezométri$c a$ (correspondientes a variaciones de sección en tubería), al incluir sólo magnitudes directamente medibles, se comparan con mediciones directas de longitud y de sección, no fundamentadas en leyes experimentales (las hipótesis justificadoras de estas mediciones - relativas a rigidez, coincidencia y combinación física de reglas - no son leyes mecánicas).

Además, y esto es muy importante, esta reestructuración lógica de la contrastación es perfectamente generalizable. Tiene sentido comparar una predicción y una medición sólo si lo son de una misma magnitud; la presión en el ejemplo considerado, o $M$ en un caso general. La predicción y la medición tienen, entonces, las formas genéricas: " $M=$ $f\left(m_{i}\right)$ " y " $M=g\left(m_{j}\right)$ ", respectivamente, en las cuales $m_{i}$ y $m_{j}$ representan magnitudes medibles de modo directo. De esas formas se deduce inmediatamente: " $f\left(m_{i}\right)=g\left(m_{j}\right)$ ", predicción de magnitudes $\left(m_{i}, m_{j}\right)$ que se miden de modo directo. En consecuencia, siempre es posible reformular 
la contrastación experimental según el siguiente esquema general:

Premisas de predicción $\rightarrow M=f\left(m_{i}\right)$

Premisas de medición $\rightarrow M=g\left(m_{j}\right)$

$$
M=f\left(m_{i}\right) \text { y } M=g\left(m_{j}\right) \rightarrow f\left(m_{i}\right)=g\left(m_{j}\right) .
$$

Premisas de predicción y de medición $\rightarrow f\left(m_{i}\right)=$ $g\left(m_{j}\right)$.

Esta remodelación de la contrastación experimental como una derivación de predicciones de efectos directamente medibles (de alturas piezométricas en lugar de presiones) tiene dos consecuencias inmediatas. En primer lugar, desaparece la impresión de circularidad porque esos efectos se comprueban con mediciones directas (que utilizan teoría, hipótesis sobre propiedades físicas de reglas, pero no se fundamentan en leyes de las teorías contrastadas). Y segundo, desde este nuevo punto de vista, las leyes que "se repetian" en la predicción y en la medición (cuando eran consideradas del modo usual) intervienen ahora doblemente para generar la predicción observable. Como consecuencia de ello, ante posibles refutaciones, tendrán más posibilidades de ser modificadas. La apariencia de autoprotección se transforma asi en su opuesta, de mayor riesgo ante la experiencia. Esto va mucho más acorde con las evaluaciones de validez o verdad que se producen de hecho en la ciencia.

\section{Conclusión}

La reestructuración lógica de la contrastación experimental permite comprender, en primer lugar, por qué los científicos no necesitan tomar decisiones especiales en las contrastaciones experimentales con circularidad. Hasta tal punto 
no las necesitan que lo más frecuente en ellos es la ausencia de percepción de tal circularidad. Ante todo, porque la labor científica es colectiva y unos se aprovechan del trabajo de otros: éstos construyen buenos medidores y aquéllos los usan para contrastar. Y, después, porque en toda contrastación teoría-experimento hay predicción de efectos observables o directamente medibles. Esto es así también en los casos de circularidad extrema, frecuentes sobre todo en la física, en los que la misma teoría generadora de la predicción interviene de modo esencial en la fundamentación de la medición contrastadora (piénsese en Torricelli, descubridor de la ley de la hidrostática y, simultáneamente, constructor de los manómetros, basados en, y contrastadores de, dicha ley). En definitiva, la experiencia siempre tiene efecto controlador, independientemente de las posibles circularidades en los principios teóricos o en las leyes.

En segundo lugar, no sólo se disuelve la circularidad al reestructurarse las contrastaciones del modo indicado. Además de ello, se produce un tremendo aumento del "holismo o globalismo en la generación de predicciones". La predicción de efectos observables, en el nuevo esquema, se deduce de muchas más premisas que la predicción de magnitudes indirectamente medibles en el esquema usual. La nueva predicción se deduce de la unión de las antes denominadas "premisas de la predicción" y "premisas de la medición". En conclusión, los dos rasgos básicos de la contrastación experimental, "globalismo de las predicciones" y "carga teórica de las mediciones", contribuyen a una misma problemática metodológica, de la cual sí son muy conscientes los científicos, la resumida en la expresión “problema de Duhem".

Por último, pero no menos importante, desde que Hanson (en Patterns of Discovery, (1958)) y Kuhn (en The Structure of Scientific Revolutions, (1962)) consiguieron 
extender la tesis de la "carga teórica de la medición" (y recuperaron a Duhem), el análisis detallado e insuficiente de las contrastaciones experimentales ha conducido a popularizar entre los filósofos la imagen de que la experiencia tiene un papel relativamente débil en las ciencias naturales. Del empirismo ingenuo positivista se ha pasado a un teoricismo supuestamente crítico pero, en el fondo, ingenuo. Se ha llegado a extremos, como el de la concepción estructuralista, iniciada por Sneed (1971), en la que se reformulan las teorías físicas de un modo bastante forzado para resolver el problema planteado por los "conceptos de un teoría $T$ cuyas mediciones requieren siempre usar leyes de esa misma teoría $T$ ". Estos "conceptos $T$-teóricos" no plantean, vistas las discusiones anteriores, ningún problema especial. Una teoría física puede intervenir de modo pleno en el fundamento de su contrastación, como la mecánica de fluidos en el caso del efecto Venturi, sin que resulte disminuida, por ello, la posibilidad de ser afectada por el veredicto experimental. Pero el exceso de teoricismo no es un defecto exclusivo de los estructuralistas. Todos los filósofos de la ciencia actuales hemos desarrollado nuestro trabajo en un atmósfera intelectual "teoricista ingenua". $\mathrm{Y}$ todos estamos obligados a modificar seriamente nuestras concepciones respecto al poder controlador de la experiencia. 
Apéndice I. Ley irrotacional de Bernoulli

(Ley del movimiento)

$$
\begin{aligned}
& \frac{d \vec{F}_{\text {ext. }}}{d V}+\eta \nabla^{2} \vec{v}+\left(\eta+\eta^{\prime}\right) \nabla(\nabla \vec{v})-\nabla P=\rho\left((\vec{v} \cdot \nabla) \vec{v}+\frac{\partial \vec{v}}{\partial t}\right) \\
& -\rho \nabla g h+\eta \nabla^{2} \vec{v}+\left(\eta+\eta^{\prime}\right) \nabla(\nabla \vec{v})-\nabla P=\rho\left((\vec{v} \cdot \nabla) \vec{v}+\frac{\partial \vec{v}}{\partial t}\right) \\
& \frac{d \vec{F}_{\text {ext. }}}{d V}=-\rho \nabla g h \\
& \eta=0 \text { y } \eta^{\prime}=0
\end{aligned}
$$

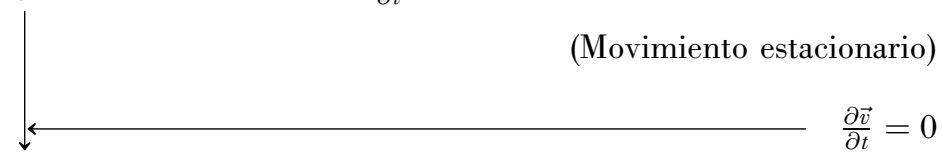

$\nabla P+\rho \nabla g h+\rho(\vec{v} \cdot \nabla) \vec{v}=0$

(Teorema matemático)

$$
(\vec{A} \cdot \nabla) \vec{A}=\nabla \frac{1}{2} A^{2}+(\nabla \times \vec{A}) \times \vec{A}
$$

$\nabla P+\rho \nabla g h+\rho \nabla \frac{1}{2} v^{2}+\rho(\nabla \times \vec{v}) \times \vec{v}=0$

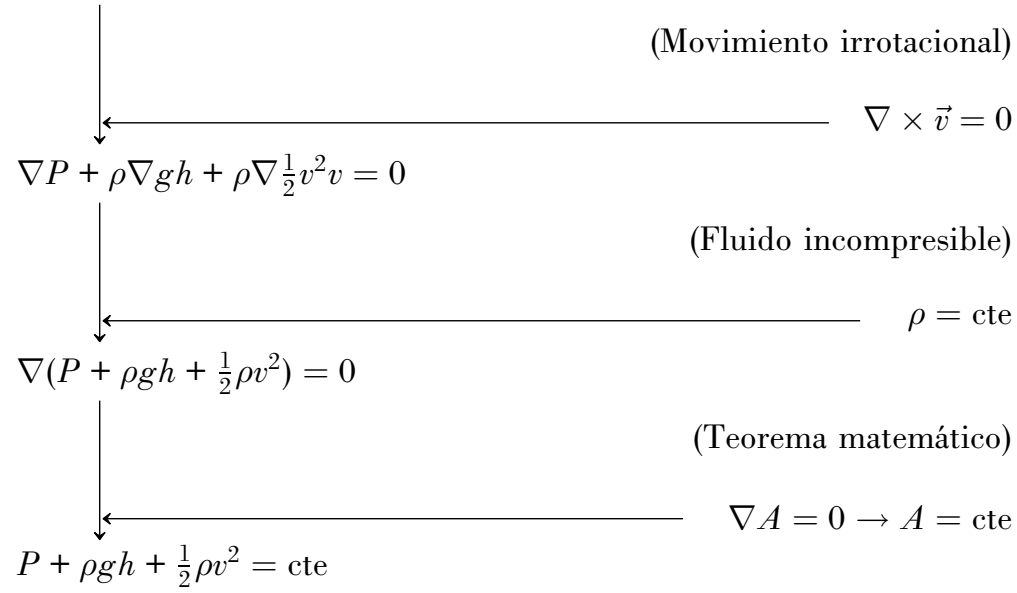

(Bernouilli irrotacional) 


\section{Apéndice II. Ley de Torricelli}

(Ley del movimiento)

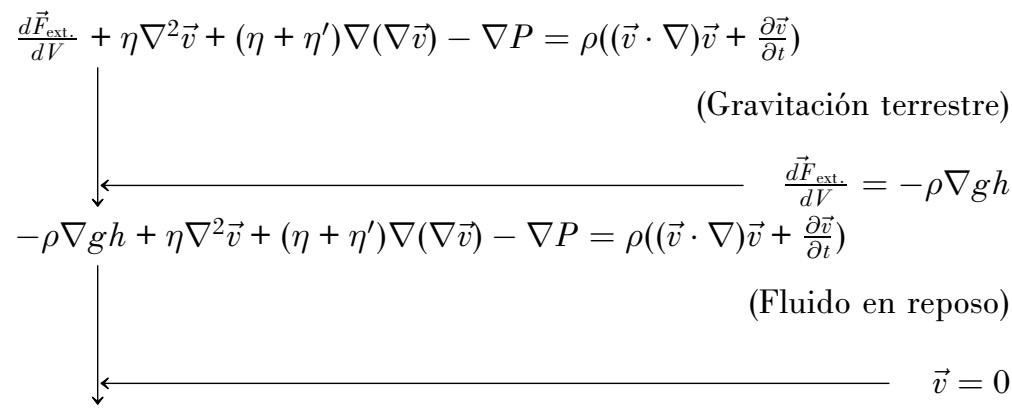

$\nabla P+\rho \nabla g h=0$

(Fluido incompresible)

$\nabla(P+\rho g h)=0$

$\rho=$ cte

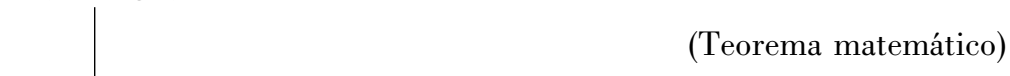

$\nabla A=0 \rightarrow A=$ cte

$P+\rho g h=$ cte

(Ley de Torricelli)

(La ley del movimiento utilizada en este apéndice y en el anterior expresa, para un elemento diferencial de volumen de fluido:

densidad de fuerza externa + densidad de fuerza interna de viscosidad (tangencial) + densidad de fuerza interna de presión (normal)

= densidad de masa $\cdot$ aceleración,

donde la aceleración resulta de aplicar el teorema "regla de la cadena" a su definición: $a=d v(x, y, z, t) / d t$. 
Apéndice III. Ecuación de continuidad

(Conservación de la masa)

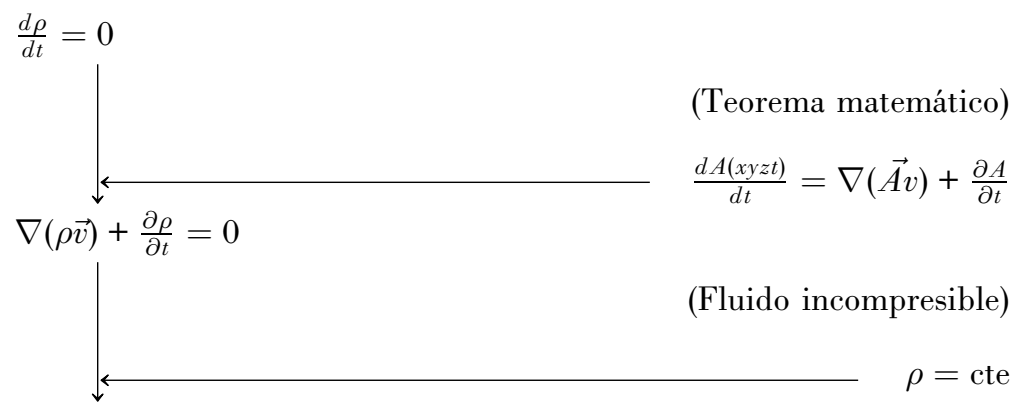

$\nabla \vec{v}=0$

(Teorema matemático)

$\int \nabla \vec{A} d V=\oint \vec{A} \cdot \overrightarrow{d S}$

$\oint \vec{v} \cdot \overrightarrow{d S}=0$

(Tubo de corriente)

$\int \vec{v} \cdot \overrightarrow{d S}=$ cte

$\oint \vec{v} \cdot \overrightarrow{d S}=-\int_{S_{\mathrm{ent}}} \vec{v} \cdot \overrightarrow{d S}+\int_{S_{\mathrm{sal}}} \vec{v} \cdot \overrightarrow{d S}$

(Movimiento irrotacional)

$\nabla \times \vec{v}=0 \rightarrow \vec{v}=$ cte en $S$ del tubo

$v S=$ cte

(Ecuación de continuidad, incompresible e irrotacional en tubos de corriente) 


\section{BIBLIOGRAFÍA}

Bunge, M., 1967, Scientific Research, vol. II, The Search for Truth, Springer Verlag, Berlín/Heidelberg/Nueva York.

— , 1973, Philosophy of Physics, Reidel, Dordrecht/Boston.

Duhem, P., 1905, La théorie physique, son objet et sa structure, Chevalier/Rivière, París.

Hanson, N.R., 1958, Patterns of Discovery, Cambridge University Press.

Kuhn, T.S., 1962, The Structure of Scientific Revolutions, University of Chicago Press.

Sneed, J.D., 1971, The Logical Structure of Mathematical Physics, Reidel, Dordrecht/Boston.

Recibido: 19 de marzo de 1994 


\section{SUMMARY}

The experimental test of scientific laws has two essential characteristics: (a) the holism of predictious generation (the predictions are deduced from sets of laws and not from individual laws) and (b) the theoretical weight of measurements (the measurements are based on laws). The former aspect brings about "Duhem's problem": if a prediction is refuted, it is necessary to decide which of its different premises must be modified. Point (b) above implies circularities in experimental test that can be extreme: the same laws may be part of the deduction of a prediction and, simultaneously, of the fundaments of the prediction that allows to verify it. The analysis of one of such cases, the Venturi effect, reveals that circularity does not protect the theories of experimentation and, furthermore, that its methodological effects reduce to reinforce holism, thus increasing "Duhem's problem". 